\title{
ESTRATEGIAS EN LA CONSTRUCCIÓN DE UN PROTOTIPO COMO MODELO INTEGRAL EN LA GESTIÓN INVESTIGATIVA ORIENTADO HACIA EL ESQUEMA DE NEGOCIO
}

\author{
StRATEGIES IN THE CONSTRUCTION OF A PROTOTYPE AS \\ AN INTEGRAL MODEL IN RESEARCH MANAGEMENT ORIENTED \\ TOWARD THE BUSINESS SCHEME
}

\section{${ }^{1}$ Ochoa G. Nancy E, ${ }^{2}$ Cruz B. Ingrid M, ${ }^{3}$ Gil Celio E., ${ }^{4}$ Silva Chaves Cristian Camilo, ${ }^{5}$ Sara Ketherine Grajales B., ${ }^{6}$ Leidy Lorena Vergara Vargas, ${ }^{7}$ Perea Díaz María Paula, ${ }^{8}$ Alonso Páez}

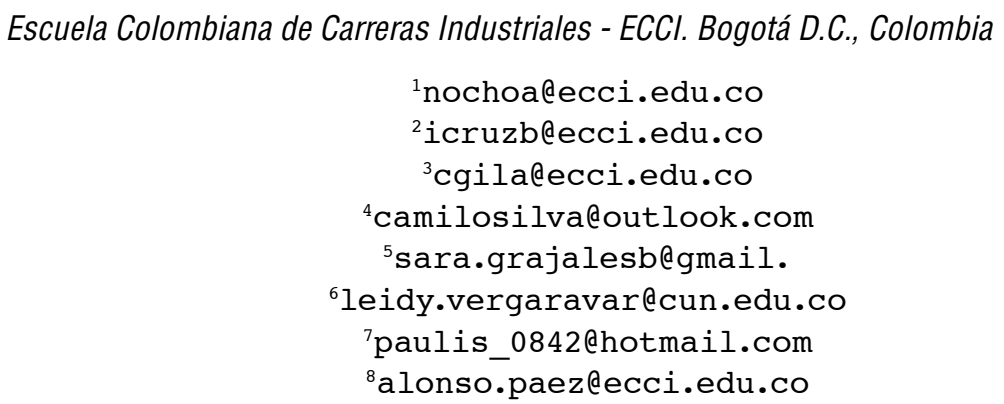

\section{RESUMEN}

Este artículo muestra los resultados del proyecto Geinve v1.0, el cual busca fortalecer los procesos automatizados de la universidad $\mathrm{ECCl}$, con el apoyo de su grupo de investigación Sistema de Gestión Científica y Tecnología, Sigcienty, y los semilleros Mente Viva y Aprovechando la Web. Además se responde a la pregunta de investigación: ¿cuáles son las estrategias para la construcción de un prototipo inicial para un modelo integral en la gestión investigativa en la Universidad ECCl, orientado hacia el esquema de negocio? Dando alcance a la estructura organizacional de la Universidad y a la Gerencia de Proyectos de la Vicerrectoría de Investigación, se tomó como base para el desarrollo de este trabajo, la metodología RUP, con el acompañamiento del lenguaje del modelo unificado-UML y la metodología Proyect Management Body of Knowledge -PMBOK-, con el fin de administrar y controlar la gestión de los proyectos de investigación desde el área de negocios y las especificaciones métricas 
de un sistema de información (software), respectivamente. En el avance de dicha gestión se implementó un prototipo funcional en línea a través de un sitio web, como método de prueba del avance de dichas metodologías, logrando dar una iniciativa al interior de la Universidad ECCI sobre la organización y valorización de los productos de investigación de la comunidad científica y académica en la ciudad de Bogotá, Colombia.

Palabras clave: integralidad, metodología, negociación, prototipo, sistemas de información.

\section{Abstract}

This article shows the results of GEINVE v1.0 project contributing to the strengthening of the automated processes of the ECCl university with the support of its hotbeds groups Mind taking advantage of the web and viva, led by the research group System of Scientific Management and Technology SIGCIENTY. Achieving answer your research question ¿What are the strategies for the construction of an initial prototype for a model in Investigativa Integral Management at the University ECCI oriented business model ?. Overtaking the organizational structure of the University and project management in their research Vicerrectorí. The methodology used is given through a schematic triangle as was the Rational Unified Process -RUP, with accompanying Modeling Language Unified -UML and the methodology Project Management Body of Knowledge -PMBOK, in order to manage and control the management of research projects from the business area and metrics specifications of an information system (software ) respectively. In advance of such management a functional prototype online was implemented through a web site as a method of testing the progress of these methodologies, succeeding in giving an initiative within the ECCI University on the organization and enhancement of research products the scientific and academic community in the city of Bogotá, Colombia.

Words Keys: integrity, information systems, methodology, negotiation, prototype.

\section{INTRODUCCIÓN}

En este momento, la gran mayoría de las universidades colombianas invierten en sistemas de información como repositorios para llevar el control y manejo de la producción investigativa en sus grupos y semilleros de investigación. No obstante, la mayoría son sistemas muy tradicionales y muy pocos, por no decir que ninguno, evalúan realmente la importancia y ganancia de la producción científica al interior de la Institución [1].

Colciencias, Departamento Administrativo de Ciencia, Tecnología e Innovación, es el ente encargado en Colombia de promover las políticas públicas para fomentar la Ciencia, Tecnología e Innovación (CT+l). Las actividades alrededor del cumplimiento de su misión implican concertar políticas de fomento relacionadas con la producción de conocimientos, construir capacidades para $\mathrm{CT}+\mathrm{l}$ y propiciar la circulación y uso de los mismos para el desarrollo integral del país y el bienestar de los colombianos [2].

Vale aclarar, que la mayoría de las instituciones de educación superior en Colombia se han 
limitado a cumplir estrictamente lo que Colciencias exige en su Modelo de Medición de Grupos. Sin embargo, es importante ir más allá y tomar este modelo como base primordial, primero para evaluar al interior de las instituciones educativas la relevancia de su producción científica como aporte a las mallas curriculares, a la proyección social y a las líneas de investigación como tal y segundo, para tener en cuenta la visión nacional e internacional de dicha producción acorde con la globalización tecnológica y económica [3].

La gestión investigativa al interior de la Universidad ECCI se realiza gracias al desarrollo de propuestas de investigación de sus grupos semilleros y jóvenes investigadores, por medio de convocatorias internas anualmente o por la participación en convocatorias abiertas de alguna institución externa, entre otros. En ciertos casos, logra hacer sinergias de estas convocatorias con otros grupos, quedando este desarrollo muy interno a la institución. Estos proyectos, en algunas oportunidades, como sucede en muchas IES, se quedan solo en documentos con publicaciones en artículos y uno que otro capítulo de libro [1], perdiendo gran relevancia en los aportes a la comunidad científica, como indicador de crecimiento al país y al proceso de internacionalización que la institución pueda aportar como productos de alta calidad [3].

Acorde con el contexto anterior, surge la necesidad, con el apoyo de Colciencias a los grupos semilleros, de realizar este proyecto Geinve v1.0, con el fin de abrir un camino hacia la valoración de la producción científica, académica y tecnológica como aporte al fortalecimiento de la Institución Educativa a través de la construcción de nuevo conocimiento en la comunidad.

Teniendo en cuenta la pregunta de investigación que se planteó la Universidad, ¿cuáles son las estrategias y elementos en la construcción del prototipo inicial para un Modelo Integral en la Gestión Investigativa de la Universidad ECCI orientado hacia el esquema de negocio?, el objetivo general del estudio se centra en identificar las estrategias y los elementos en la construcción del prototipo inicial para dicho Modelo orientado hacia el esquema de negocio, con base en la metodología unificada RUP, el apoyo del lenguaje UML y la metodología PMBOK.

Se logra conocer algunas estrategias y ciertos elementos para dar inicio al esquema del prototipo como soporte al modelo integral, bajo la aplicación de las metodologías recomendadas.

\section{Antecedentes}

Se toma como base estudios y proyectos realizados en Latinoamérica, Europa y Norteamérica con base en metodologías RUP/PMBOK, a través de la implementación del Lenguaje UML y el método de prototipo para lograr evaluar dichas metodologías: PRO.

\section{A. Estudio en Colombia}

1) Proyecto. Plantillas y artefactos: personalización de RUP para proyectos académicos de desarrollo de software. [4]

2) Objetivo. Apoyar la metodología personalizada para los proyectos académicos de desarrollo de software, realizando sus respectivas plantillas y artefactos para la ejecución de dicho framework, a través de las disciplinas de gestión de proyectos, modelado empresarial, requisitos, análisis y diseño, implementación, pruebas, despliegue y gestión del cambio, teniendo como referentes las metodologías estándar RUP, CMMi DEV y PMBOK.

3) Enfoque. Hacer uso de los flujos de trabajo de cada disciplina, en la ejecución de cada tipo de proyecto académico, ya definidos en el framework, para realizar sus plantillas y artefactos. Determinar los roles y las responsabilidades de los encargados del proceso de software 
para cada disciplina, objeto de este proyecto, de acuerdo con cada tipo de proyecto académico, para apoyar el desarrollo de los artefactos ejemplo. Construir las plantillas y artefactos del framework.

4) Resultados. La creación del software EDU que establece la metodología unificada RUP, para ejecutar los proyectos académicos de desarrollo de software, que se proponen en las asignaturas que pertenecen al área de Ingeniería de Software de la Universidad EAFIT, tanto de programas de pregrado, como de posgrado.

\section{B. Estudios latinoamericanos}

1) Proyecto. El modelado del negocio utilizando la metodología Rational Unified Process (RUP) [5].

2) Objetivo. Destacar el modelado del negocio, para la exitosa ejecución de proyectos intensivos en desarrollo de software, utilizando la metodología del Proceso Unificado de Desarrollo de Software (RUP) y presentando un enfoque prescriptivo para estandarizar las mejores prácticas de la ingeniería de software.

3) Enfoque. Implementar mecanismos en las organizaciones que permitan escalar el software con altos niveles de estándares a través de la metodología RUP, basada en el modelamiento del negocio a la satisfacción del cliente.

4) Resultados. El modelado del negocio a través del proceso de ingeniería de software permitiendo, a lo largo del tiempo, asegurar una buena producción de software de alta calidad que satisfaga la necesidad de un usuario final dentro de un tiempo y presupuesto previsibles. Esta investigación se realizó en Perú.

\section{Estudios norteamericanos}

1) Proyecto. Software educativo para la enseñanza-aprendizaje del Psicodiagnóstico de Rorschach [6].
2) Objeto de estudio. Perfeccionar los procesos de enseñanza y aprendizaje de la técnica proyectiva Psicodiagnóstico de Rorschach por medio de la creación de un software educativo que implementaría la metodología RUP a través del apoyo del lenguaje UML, en el que se identificaría el proceso de control y manipulación de la información obtenida.

3) Enfoque. Por medio de un muestreo estratificado por año académico y vía de ingreso, con una muestra de 50 estudiantes y 10 profesores de la Filial de Ciencias Médicas, Lidia Doce, de Sagua la Grand, el estudio constó de cuatro etapas: exploratoria, planificación, ejecución y evaluación.

4) Resultado. Se diagnosticaron las necesidades educativas. Con la aplicación de la prueba estadística no paramétrica Anova de un factor, y la información obtenida, se triangularon los resultados. Con esta metodología se documentó la ingeniería del software educativo Sedror. El sistema fue elaborado con la herramienta Visual Adobe Director 11.5. Lugar: Sagua la Grande. Villa Clara. Cuba. Universitaria de Ciencias Médicas "Lidia Doce Sánchez".

\section{Estudios europeos}

1) Proyecto. Modelado de procesos y desarrollo de sistemas software: integración entre UML y EPC. [7].

2) Objeto de estudio. Los objetivos de Unified Modeling Language-UML (Lenguaje Unificado de Modelado) y de la Event-driven Process Chain-EPC, (Cadena de Procesos guiada por Eventos) están bien diferenciados: mientras que UML se enfoca al diseño de sistemas de información (SI), las EPCs se emplean para el modelado de procesos de negocios BPM, dentro de la metodología ARIS.

3) Enfoque. No obstante, es evidente la relación entre ambas técnicas: por una parte, un correcto diseño de si debe basarse en los requisitos 
definidos por el modelo de procesos de negocio $y$, por otra, las mejoras de los procesos existentes que a menudo deben llevarse a cabo mediante el desarrollo o modificación de los sistemas de información que soportan dichos procesos.

4) Resultados. Creación de la plataforma gráfica para visualizar, especificar, construir y documentar los artefactos pertenecientes a un sistema, cubriendo las partes conceptuales (funciones del sistema) y en principio también los procesos de negocios y los elementos concretos (clases escritas en un lenguaje de programación específico, esquemas de bases de datos, componentes software reutilizables). Lugar: Sevilla España.

\section{Materiales}

Normatividad de las metodologías PMBOK, RUP y UML. Método de Prototipo PRO para la evaluación y análisis de la metodología. Uso de herramientas libres para el diseño del prototipo como: Java Script y JSP, con el soporte de una base de datos MySQL puesto que con ellos se logró concretar la viabilidad del proyecto y llegar a los resultados esperados. El diseño de la página web se hizo a través de la herramienta IDE - NETBEENS ver. 8.2, en razón a su facilidad de manejo. Además, porque cumple perfectamente con el objetivo de diseñar portales con aspecto profesional puesto que soporta gran cantidad de tecnologías como: hojas de estilo y capas, Java script para crear efectos e interactividades, tales como, el caso del estudio, y, finalmente, la inserción de archivos multimediales.

Se utilizó Java Servet Pages (JSP), en razón a que permite la creación de aplicaciones en tres capas (base de datos, negocios y diseño); así mismo, su modo de funcionamiento permitió construir una interactividad con la web (internet), logrando una aplicación abierta para los actores de la investigación, en este caso, de la Universidad $\mathrm{ECCl}$.

\section{Metodologia}

El prototipo Geinve_v1.0 se diseñó en cuatro fases:

\section{A. Fase I.}

Diseño y universo de la investigación (instrumentos de evaluación DOFA, encuesta)

\section{B. Fase II.}

Metodología RUP. Inicio. Determinó la versión del proyecto y su acta de puesta en marcha. Elaboración. Determinó la arquitectura óptima del sistema con la ayuda de algunas herramientas libres bajo la licencia GNU. Construcción. Capacidad operacional inicial del sistema, permitiendo reforzar los procesos de la metodología de negocio con la del software. Transmisión. Obtención del reléase del proyecto, con el fin de lograr instalarlo en los servidores de la Universidad $\mathrm{ECCl}$ e iniciar su etapa de puesta en marcha y de madurez, estableciendo los puntos fuertes y débiles, para buscar la mejora del sistema.

\section{Fase III.}

Metodologia PMBOK para rescatar el inicio del esquema de negocio en el diseño del prototipo Geinve, v1.0, como: acta de inicio, planeación, ejecución, control, riesgos y cierre. Para tal fin, se crea una estructura de carpetas con el fin de recoger la información de algunos proyectos como prueba para dicho prototipo como es proyectos de investigación; grupos, jóvenes y semilleros y repositorio documental [5].

\section{Fase IV.}

Aplicación del método del prototipo PRO en las metodologías utilizadas.

\section{Resultados}

Este estudio permitió en gran parte dilucidar los vacíos existentes en cuanto a normativas y 
procedimientos, lo que facilitó y garantizó un trabajo coordinado entre las distintas partes. De esta forma, se pudieron incorporar los procesos y fases de las metodologías PMBOK y RUP, con el uso del lenguaje de casos UML, para visualizar y mejorar los puntos fuertes y débiles alcanzados en cada artefacto de las metodologías. Además, se construyó el prototipo Geinve_v1.0 a la medida para dar inicio a la gestión investigativa de la Universidad. Este prototipo es un producto de software propiamente tal, susceptible de ser explotado en un ambiente real, bajo una plataforma tecnológica de MySQL y JSP.

\section{A. Matriz DOFA}

Para construir la matriz DOFA, se realizaron algunas entrevistas a los profesionales de la Vicerrectoría de Calidad y Acreditación y la Vicerrectoría de Investigación, y se utilizaron, como apoyo, documentos oficiales publicados en la página institucional de la Universidad, http:/ecci. edu.co. En la tabla 1 se presenta una descripción consolidada de las debilidades y fortalezas encontradas durante el proceso de observación y la entrevista a los funcionarios encargados, al igual que en el análisis de la documentación existente en su momento. Esto permitió establecer la lista de posibles riesgos a corto y mediano plazo.

TABLA I.

Matriz Dofa del Estudio

\begin{tabular}{|c|c|c|}
\hline Factores Internos & $\begin{array}{l}\text { FORTALEZAS (F) } \\
\text { F1: Investigadores con capacidad de } \\
\text { generar conocimiento científico y hacer } \\
\text { aportes a la comunidad científica (Em- } \\
\text { poderamiento del trabajo). } \\
\text { F2: Posicionamiento en la comunidad } \\
\text { científica } \\
\text { F3: Tendencia al crecimiento como } \\
\text { Universidad vs empresa / Empresa vs } \\
\text { Universidad. }\end{array}$ & $\begin{array}{l}\text { DEBILIDADES(D) } \\
\text { D1: Paradigmas acerca del desa- } \\
\text { rrollo de formas I+D+i. } \\
\text { D2: Mala comunicación entre los } \\
\text { stakeholders. } \\
\text { D3: Recursos compartidos en } \\
\text { entre el área de producción e } \\
\text { investigación y desarrollo. } \\
\text { D4: Cambio constante de prioriza- } \\
\text { ción en el desarrollo de la investi- } \\
\text { gación innovación. } \\
\text { D6: Ineficiente control y vigilancia } \\
\text { de los procesos. }\end{array}$ \\
\hline $\begin{array}{l}\text { OPORTUNIDADES (0) } \\
\text { 01: Tendencia internacional de utilizar } \\
\text { las herramientas de gestión de pro- } \\
\text { yectos para obtener mejores resulta- } \\
\text { dos (Benchmarking). } \\
\text { 02: Compromiso de los proveedores } \\
\text { con el desarrollo de productos nuevos } \\
\text { (Outsourcing). }\end{array}$ & $\begin{array}{l}\text { Estrategias(F0) } \\
\text { - Incentivar capacitación a los actores } \\
\text { de la investigación referente a gestión } \\
\text { de proyectos. } \\
\text { Comunicación constante con los ge- } \\
\text { rentes de empresa y producción sobre } \\
\text { lo referente al desarrollo del proyecto. }\end{array}$ & $\begin{array}{l}\text { Estrategias (D0) } \\
\text { - Utilizar las herramientas de ges- } \\
\text { tión de proyectos: gestión de la } \\
\text { comunicación, gestión del alcan- } \\
\text { ce, control de cambios, gestión } \\
\text { del tiempo y gestión de riesgos } \\
\text { para obtener mejores resultados. }\end{array}$ \\
\hline $\begin{array}{l}\text { AMENAZAS (A) } \\
\text { A1: Cambio en las regulaciones para } \\
\text { la obtención de productos científicos } \\
\text { nuevos por parte del ente guberna- } \\
\text { mental. } \\
\text { A2: Lanzamiento anticipado por parte } \\
\text { de la competencia de un producto } \\
\text { similar. }\end{array}$ & $\begin{array}{l}\text { Estrategias (FA) } \\
\text { - Consulta y búsqueda de información } \\
\text { constante referente a regulaciones por } \\
\text { el ente gubernamental. } \\
\text { - Cumplir con los tiempos establecidos } \\
\text { en el cronograma de actividades }\end{array}$ & $\begin{array}{l}\text { Estrategias (DA) } \\
\text { Realizar reuniones efectivas entre } \\
\text { los stakeholders. } \\
\text { Generar las actas de reunión y } \\
\text { cierre de la misma. } \\
\text { Diligenciar los formatos de pro- } \\
\text { ductos obtenidos. }\end{array}$ \\
\hline
\end{tabular}




\section{B. Instrumentos de evaluación (encuesta)}

En la revisión que se realizó de la literatura para este estudio, no se obtuvo un instrumento estándar validado aplicable. Sin embargo se revisaron algunos estudios como: el análisis de preguntas cerradas a docentes de universidad [8] y el esquema de investigación de mercado para estudiantes y docentes en el ámbito universitario [9], entre otros. Por lo tanto, la encuesta fue construida, en su totalidad, tomando en cuenta datos relevantes que sirvieron para tener claridad sobre el estamento que estaba participando, junto con su género y programa académico de la institución. Se creó una encuesta aplicando el proceso de validez y confiabilidad, garantizando los resultados esperados con el apoyo de siete (7) expertos, de los cuales cinco (5) fueron externos a la Institución y dos (2) internos.

Las preguntas de la encuesta son de tipo Likert con el propósito de determinar cuantitativamente posiciones específicas sobre la propuesta de presentación del diseño del prototipo para la gestión investigativa en la universidad ECCI. En la tabla 2 se observan las opciones de respuesta correspondientes a cada una de las preguntas del instrumento de evaluación, desglosadas en: totalmente de acuerdo - TA; de acuerdo - DA; ni de acuerdo ni en desacuerdo - indiferente; en desacuerdo - ED y totalmente en desacuerdo - TD, las cuales conformarán los tópicos de evaluación según la escala de Likert.

TABLA ॥

Códigos de las Respuestas del Instrumento Aplicado

\begin{tabular}{c|l}
\hline Código & \multicolumn{1}{|c}{ Opciones de respuesta } \\
\hline 5 & TA = Totalmente de acuerdo \\
4 & $\mathrm{DA}=$ De acuerdo \\
\hline 3 & $\begin{array}{l}\text { NDA = Ni de acuerdo }- \text { Ni en desacuerdo } \\
\text { (Indiferente })\end{array}$ \\
\hline 2 & ED = En desacuerdo \\
\hline 1 & TD = Totalmente en desacuerdo \\
\hline
\end{tabular}

\section{Validez y confiabilidad}

El instrumento de evaluación (encuesta) se sometió a pruebas de validez y confiabilidad [10]. Se estableció el índice de alfa Cronbach con un valor mayor a 0.86 , lo cual fue satisfactorio para la continuidad del estudio. La validez del instrumento se determinó primeramente mediante una validación aparente. Para ello, se contó con los siete (7) expertos.

\section{Resultados de los expertos}

El formato guía enviado a los expertos contaba con una pauta para evaluar de 1 a 10 la pertinencia de las dimensiones y sus ítems al propósito de la encuesta, así como también con un espacio para proponer cambios y/o sugerencias. Para determinar la validez de contenido, por medio del juicio de estos expertos, se utilizó la fórmula de Lawshe [11], quien establece un valor mínimo de razón de validez de 0,82 con siete (7) expertos, para asegurar que sea improbable que el acuerdo se deba al azar. A raíz de ello, los expertos presentaron su tabla de categorización en función de la frecuencia y la valoración de cada una de las preguntas de la encuesta. En la tabla 3 se observa el consolidado de algunas preguntas con base en el cual los expertos realizaron una valoración relevante para el instrumento como tal [11]. 
TABLA III.

Resumen Categorías Seleccionadas en Función DE LA FRECUENCIA Y LA VALORACIÓN.

\begin{tabular}{|c|c|c|c|}
\hline $\mathbf{N}$ & Rasgos/Categorías & $\begin{array}{l}\text { Valor } \\
\text { Exper- } \\
\text { tos }\end{array}$ & $f$ \\
\hline 1 & $\begin{array}{l}\text { Los productos de investi- } \\
\text { gación al interior de la uni- } \\
\text { versidad deben mejorarse } \\
\text { en cuanto a su uso y acce- } \\
\text { sibilidad a toda la comuni- } \\
\text { dad. }\end{array}$ & 7.6 & 12 \\
\hline 3 & $\begin{array}{l}\text { Se considera que los es- } \\
\text { tudiantes, en especial los } \\
\text { del programa de Ingeniería } \\
\text { de Sistemas y Tecnología } \\
\text { de Desarrollo, son piezas } \\
\text { esenciales para la cons- } \\
\text { trucción de un sitio en línea } \\
\text { que controle los proyectos, } \\
\text { grupos, repositorios y de- } \\
\text { más eventos de investiga- } \\
\text { ción. }\end{array}$ & 7.5 & 25 \\
\hline 4 & $\begin{array}{l}\text { Los productos de investi- } \\
\text { gación deben ser compati- } \\
\text { bles con la misión institu- } \\
\text { cional y los objetivos de las } \\
\text { mallas curriculares de los } \\
\text { programas académicos. }\end{array}$ & 8 & 15 \\
\hline 5 & $\begin{array}{l}\text { El apoyo a los grupos semi- } \\
\text { lleros y jóvenes investiga- } \\
\text { dores fortalece el quehacer } \\
\text { académico, investigativo y } \\
\text { científico en la universidad. }\end{array}$ & 7.5 & 11 \\
\hline 10 & $\begin{array}{l}\text { La creación de prototipos } \\
\text { funcionales para la gestión } \\
\text { investigativa permite dar } \\
\text { una visión más clara sobre } \\
\text { el alcance del sistema de } \\
\text { información que debe tener } \\
\text { la universidad. }\end{array}$ & 7.8 & 35 \\
\hline 12 & $\begin{array}{l}\text { La universidad, a través de } \\
\text { su Facultad de Ingeniería y } \\
\text { Programa de Ingeniería de } \\
\text { Sistemas, debe promover } \\
\text { la creación de software ro- } \\
\text { busto y específico para el } \\
\text { manejo de la producción de } \\
\text { los proyectos de investiga- } \\
\text { ción. }\end{array}$ & 8.3 & 10 \\
\hline
\end{tabular}

f: frecuencia
En esta encuesta, dirigida a todos los estamentos de la muestra, se obtuvo un Índice de Validez de Contenido (IVC) de 0,86, pero fueron eliminados cuatro ítems (dos en cada parte del cuestionario), ya que presentaron un valor muy inferior al mínimo de razón de validez establecido por Lawshe [11] . Con estas modificaciones, el cuestionario quedó compuesto por 13 ítems. Para las respuestas, se reafirmó el escalamiento tipo Likert con las cinco opciones propuestas y su respectivo peso acorde con la tabla 4 , donde se resalta la línea a trabajar y su respectivo procedimiento lo cual da inicio a la puesta en marcha del cuestionario (encuesta).

TABLA IV

Linea y Procedimientos Finales

\begin{tabular}{l|l}
\multicolumn{1}{|c|}{ Línea } & \multicolumn{1}{|c}{ Procediientos } \\
\hline IVC & 0.86 \\
\hline Preguntas & 13 items \\
\hline Likert & Cinco Opciones \\
\hline
\end{tabular}

\section{E. Fase de tabulación y análisis de datos}

De acuerdo con los dos puntos anteriores, se tabularon los datos obtenidos en la encuesta, con el fin de conocer la percepción de los participantes en el estudio.

En la fig. 1 se muestran los resultados obtenidos de la pregunta ¿Los productos de investigación al interior de la universidad deben mejorarse en cuanto a su uso y accesibilidad a toda la comunidad? Más de cuatro cuartas partes $(83,2 \%)$ estuvo totalmente de acuerdo o de acuerdo con este indicado, mientras que una pequeña parte $(14,8 \%)$ expresó estar en desacuerdo o totalmente en desacuerdo y una porción mínima $(0,9 \%)$ expresó no estar de acuerdo ni en desacuerdo.

Por lo anterior se concluye que la mayoría de los encuestados sabe que la Universidad genera productos de investigación, los cuales deben ser más 
accesibles a la comunidad, acorde con algunos estudios realizados en los antecedentes [3], [4].

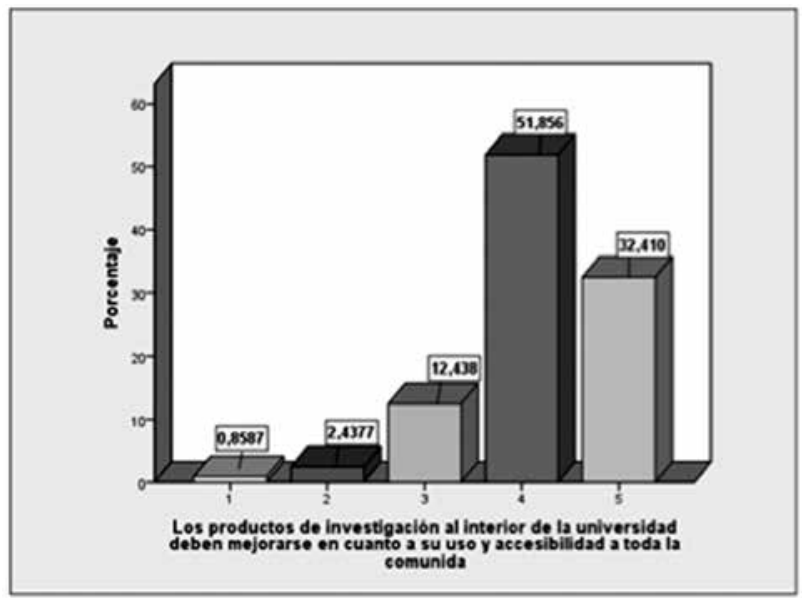

Fig. 1 Corresponde a la pregunta No. 1 del instrumento de la encuesta.

En la fig. 2 se muestran los resultados obtenidos de la pregunta ¿La universidad debe contar con un sistema adecuado para el almacenamiento, actualización y búsqueda de los productos de investigación acorde con las necesidades de la comunidad? La gran mayoría (85,3\%) estuvo totalmente de acuerdo o de acuerdo con este indicador, mientras que más de una décima parte $(13,8 \%)$ expresó estar en desacuerdo o totalmente en desacuerdo y una porción mínima $(0,9 \%)$ expresó no estar de acuerdo ni en desacuerdo.

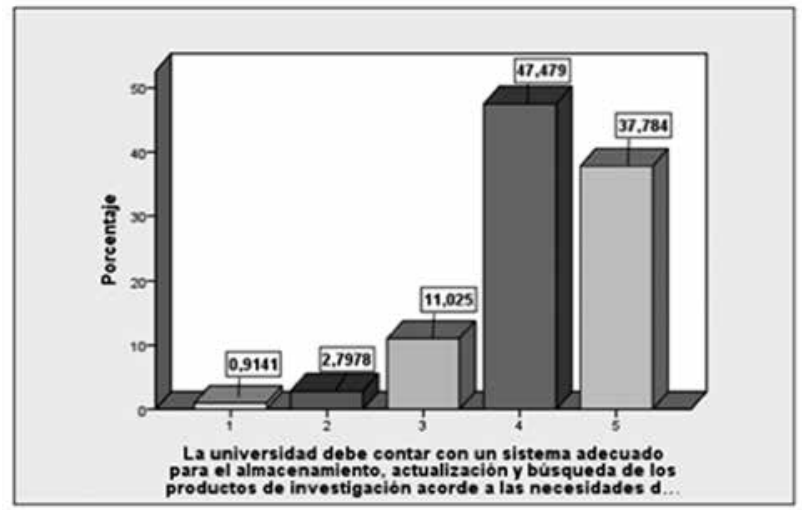

Fig. 2 Corresponde a la Pregunta No. 2 del instrumento de la encuesta

Se concluye que la mayoría de los docentes considera que los productos de investigación deben ser visibles a toda la comunidad como apoyo en propuestas o desarrollos de otros proyecto, tal como sucede con las base de datos y revistas científicas [12[, [13], [14].

En la fig. 3 e muestran los resultados obtenidos de la pregunta ¿Se considera que los estudiantes, en especial los del programa de tecnología de Desarrollo e Ingeniería de Sistemas, son piezas esenciales para la construcción de un sitio en línea que controle los proyectos, grupos, repositorios y demás eventos de investigación? Tres cuartas partes $(81 \%)$ estuvo totalmente de acuerdo o de acuerdo con este indicador; más de una sexta parte $(17,9 \%)$ expresó estar totalmente en desacuerdo o totalmente en desacuerdo y una porción mínima $(1,2 \%)$ expresó no estar de acuerdo ni en desacuerdo.

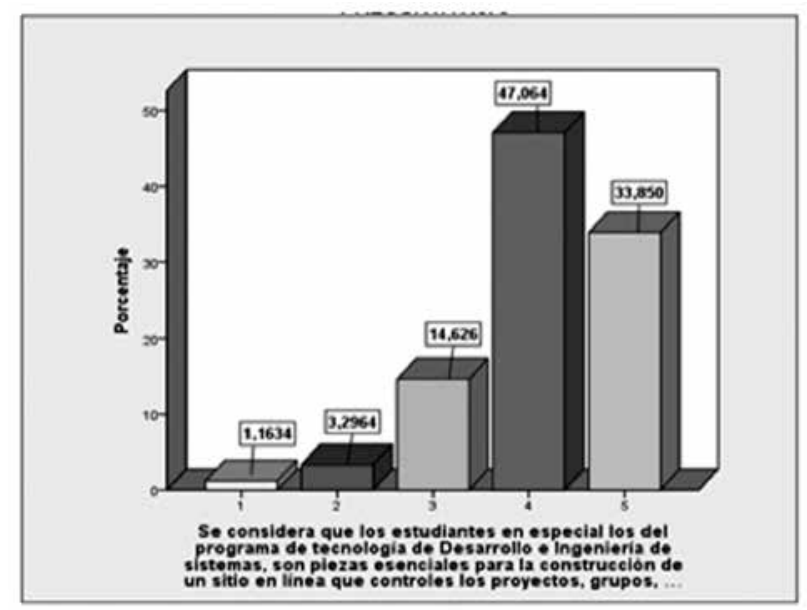

Fig. 3 Corresponde a la Pregunta No. 3 del instrumento de la encuesta.

En la fig. 4 se muestran los resultados obtenidos de la pregunta ¿Los productos de investigación deben ser compatibles con la misión institucional y los objetivos en las mallas curriculares de los programas académicos?, Más de cuatro quintas partes $(83,7 \%)$ estuvo totalmente de acuerdo o de acuerdo con este indicador; una pequeña parte $(15,5 \%)$ expresó estar en desacuerdo o totalmente en desacuerdo y una porción mínima $(0,8 \%)$ expresó no estar de acuerdo ni en desacuerdo. 


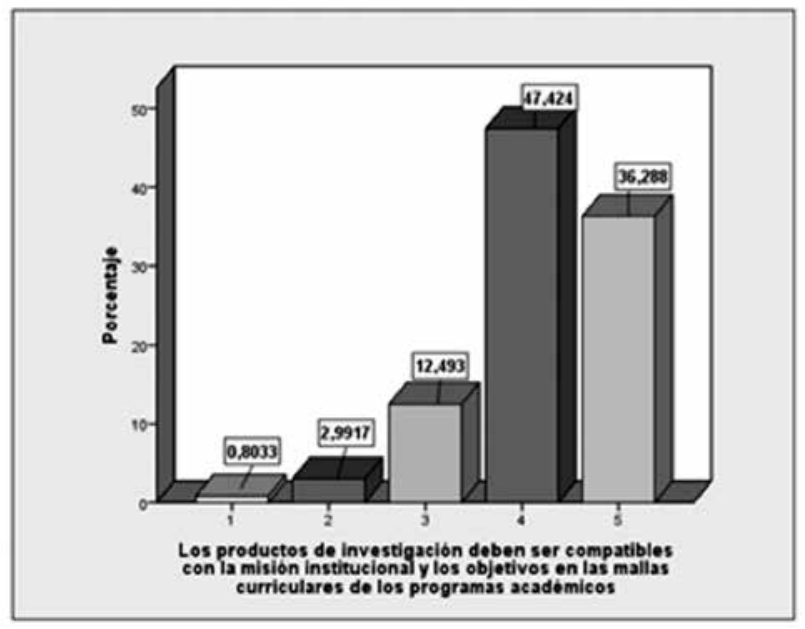

Fig. 4 Corresponde a la Pregunta No. 4 del instrumento de la encuesta.

En la fig. 5 se muestran los resultados obtenidos de la pregunta ¿El apoyo a los grupos semilleros y jóvenes investigadores fortalece el quehacer académico, investigativo y científico en la universidad? Más de dos terceras partes $(79,8 \%)$ estuvieron totalmente de acuerdo o de acuerdo con este indicador; menos de una quinta parte $(19,4 \%)$ expresó estar en desacuerdo o totalmente en desacuerdo y una porción mínima $(0,9 \%)$ expresó no estar de acuerdo ni en desacuerdo.

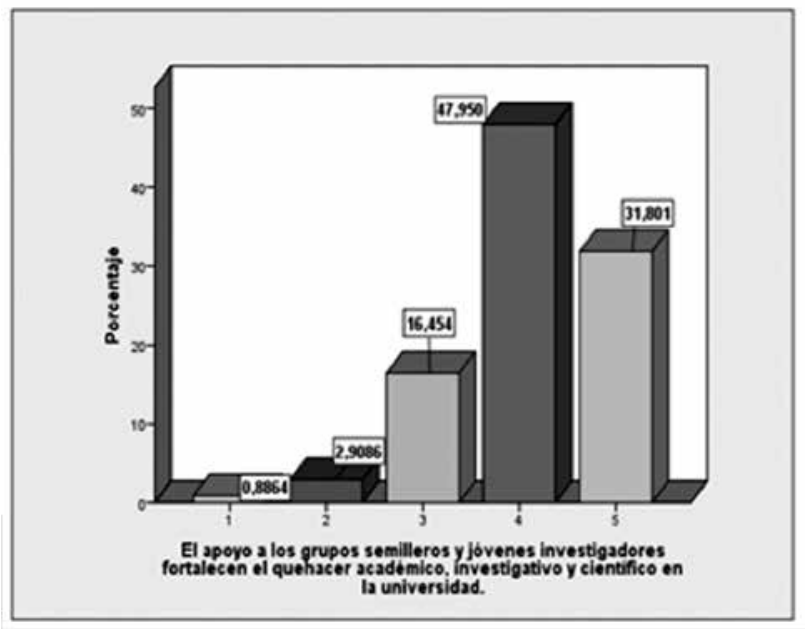

Fig. 5 Corresponde a la Pregunta No. 5 del instrumento de la encuesta.

Se resalta el apoyo de la Facultad de Ingeniería con su programa de Ingeniería de Sistemas como pieza clave, para que por medio de los proyectos de jóvenes y semilleros de investigación se contribuya al desarrollo de sistemas computacionales para el acceso a la producción científica de una manera fácil y rápida.

En la fig. 6 se muestran los resultados obtenidos de la pregunta iSe considera que diseñar aplicativos con el uso de software libre no solo minimiza los costos, sino garantiza la permanencia de su licencia al interior de la institución? La gran mayoría (84,3\%) estuvo totalmente de acuerdo o de acuerdo con este indicador; una pequeña parte (15\%) expresó estar en desacuerdo o totalmente en desacuerdo y una porción mínima $(0,8 \%)$ expresó no estar de acuerdo ni en desacuerdo.

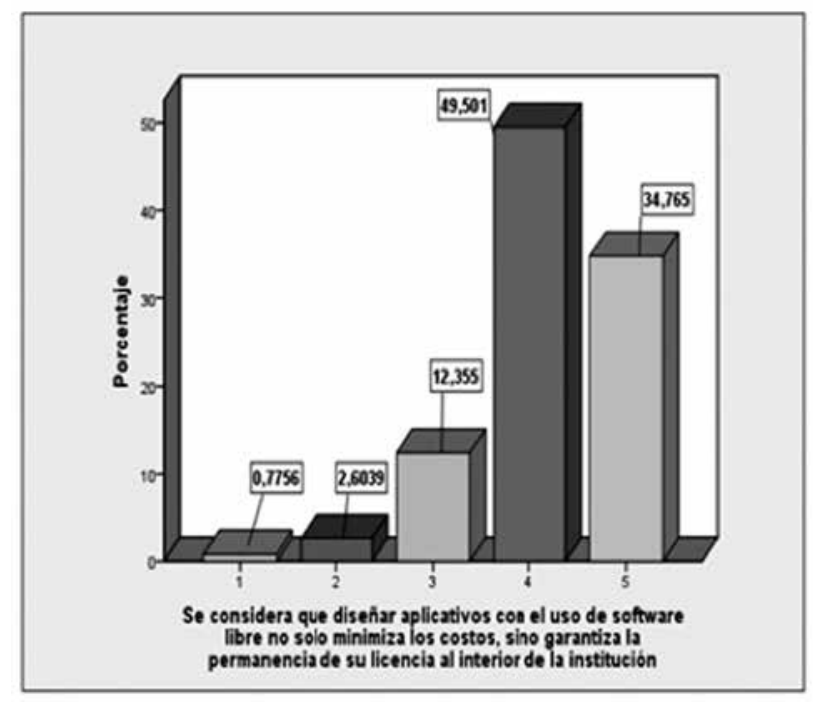

Fig. 6 Corresponde a la Pregunta No. 6 del instrumento de la encuesta.

Se concluye que la mayoría de los encuestados considera que el uso del software libre permite no solo el desarrollo a la medida, sino que fortalece el tiempo de vida útil del mismo[15], ya que su actualización se relaciona con las necesidades de la misma Institución, tal como lo recomienda MinTIC en la gobernanza TIC [16].

En la fig. 7 se muestran los resultados obtenidos de la pregunta ¿Desarrollar software propio 
al interior de la universidad asegurando su actualización inmediata, asegurando su vida útil? Un poco más de la mitad (52,9\%) estuvo totalmente de acuerdo o de acuerdo con este indicador; dos quintas partes (40,7\%) expresó estar en desacuerdo o totalmente en desacuerdo y una parte pequeña $(6,5 \%)$ expresó no estar de acuerdo ni en desacuerdo.

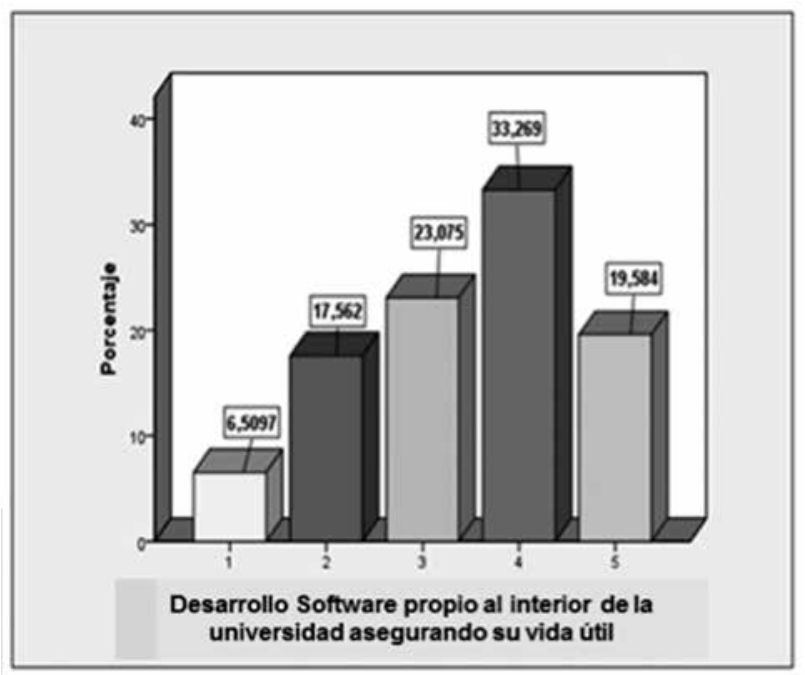

Fig. 7 Corresponde a la Pregunta No. 7 del instrumento de la encuesta.

Los resultados de esta pregunta están muy acordes con los dos anteriores, fortaleciendo el desarrollo de software propio de la institución en tiempo y espacio.

En la fig. 8 se muestran los resultados obtenidos de la pregunta ¿Se debe motivar a la comunidad universitaria en la participación de propuestas de investigación a través de un sitio web seguro y confiable para tal fin? Más de tres terceras partes $(71,1 \%)$ estuvo totalmente de acuerdo o de acuerdo con este indicador; una cuarta parte $(27,6 \%)$ expresó estar en desacuerdo o totalmente en desacuerdo y una porción mínima $(1,3 \%)$ expresó no estar de acuerdo ni en desacuerdo.

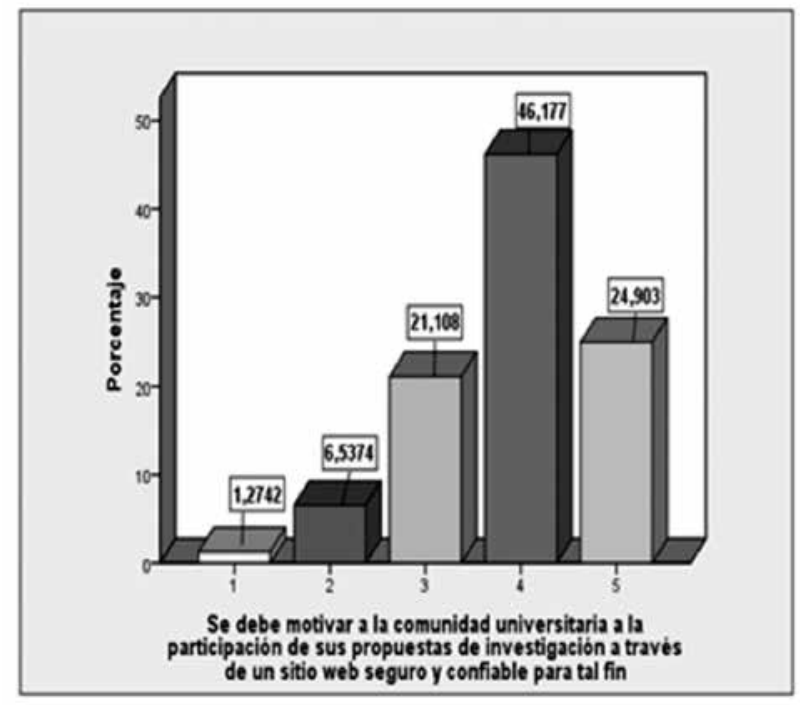

Fig. 8 Corresponde a la Pregunta No. 8 del instrumento de la encuesta

En la fig. 9 se muestran los resultados obtenidos de la pregunta ¿Los grupos de investigación deben ser piezas clave en el desarrollo de un software apropiado para la producción de investigación al interior de la universidad? Más de dos terceras partes $(67,8 \%)$ estuvo totalmente de acuerdo o de acuerdo con este indicador; una tercera parte $(30,2 \%)$ expresó estar en desacuerdo o totalmente en desacuerdo y una parte pequeña $(1,9 \%)$ expresó no estar de acuerdo ni en desacuerdo.

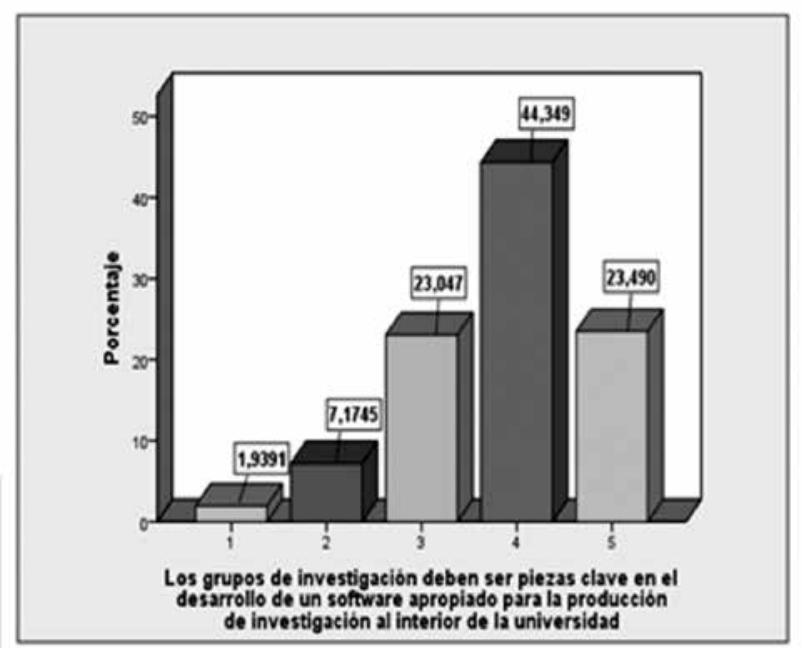

Fig. 9 Corresponde a la Pregunta No. 9 del instrumento de la encuesta 
En la fig. 10 se muestran los resultados obtenidos de la pregunta ¿La creación de prototipos funcionales para la gestión investigativa permite dar una visión más clara sobre el alcance del sistema de información que debe tener la universidad? La mayoría $(82,1 \%)$ estuvo totalmente de acuerdo o de acuerdo con este indicador; una sexta parte $(16,9 \%)$ expresó estar en desacuerdo o totalmente en desacuerdo y una porción mínima $(1,1 \%)$ expresó no estar de acuerdo ni en desacuerdo.

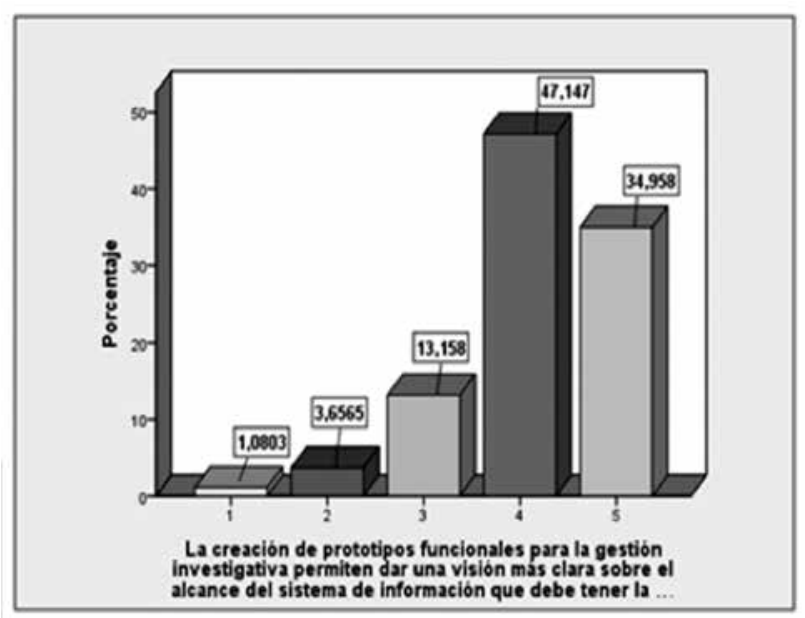

Fig. 10 Corresponde a la Pregunta No. 10 del instrumento de la encuesta

En la fig. 11 se muestran los resultados obtenidos de la pregunta ¿Se debe crear un sitio web que permite a los estudiantes, docentes y demás actores de investigación a través de unos datos de acceso ingresar a conocer todo lo referente a la producción en avance o terminada de investigación? Más de dos terceras parte $(74,4 \%)$ estuvo totalmente de acuerdo o de acuerdo con este indicador; menos de una cuarta parte $(24,5 \%)$ expresó estar en desacuerdo o totalmente en desacuerdo y una porción pequeña $(1,1 \%)$ expresó no estar de acuerdo ni en desacuerdo.

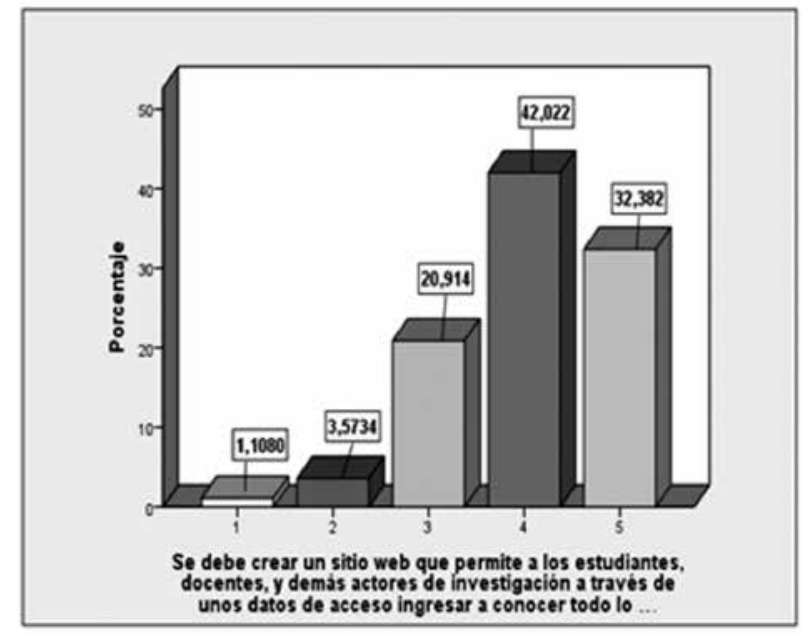

Fig. 11 Corresponde a la Pregunta No. 11 del instrumento de la encuesta

En la fig. 12 se muestran los resultados obtenidos de la pregunta ¿La universidad a través de su Facultad de Ingeniería y Programa de Sistemas, debe promover la creación de software robusto y específico para el manejo de la producción de los proyectos de investigación? Más de dos terceras partes $(65,1 \%)$ estuvo totalmente de acuerdo o de acuerdo con este indicador; una tercera parte $(33,3 \%)$ expresó estar en desacuerdo o totalmente en desacuerdo y una porción mínima $(1,6 \%)$ expresó no estar de acuerdo ni en desacuerdo.

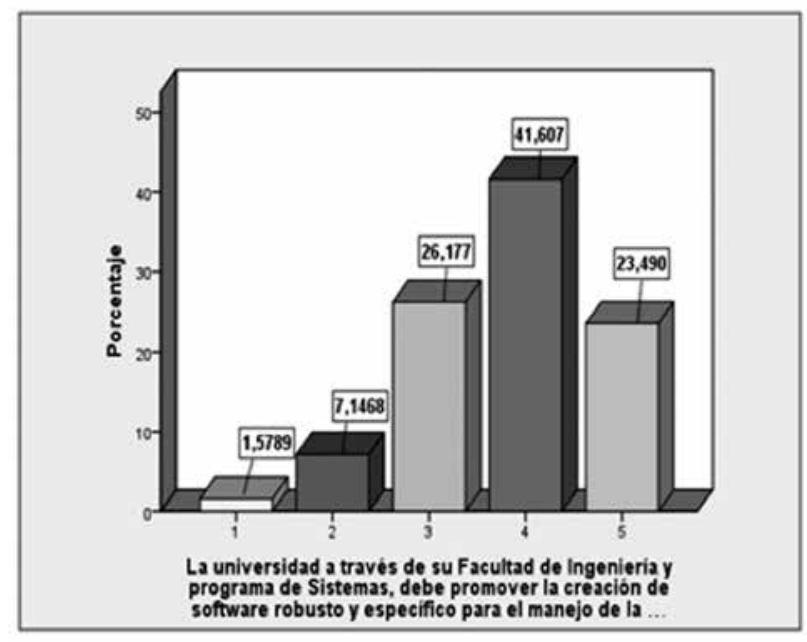

Fig. 12 Corresponde a la Pregunta No. 12 del instrumento de la encuesta 
En la fig. 13 se muestran los resultados obtenidos de la pregunta ¿Desde la Vicerrectoría de Investigación se debe promover el diseño y construcción de un sistema de información robusto que garantice en forma segura las consultas $y$ búsquedas de los proyectos y sus respectivos productos? La mitad $(58,2 \%)$ estuvo totalmente de acuerdo o de acuerdo con este indicador; menos de dos quintas partes (36,5\%) expresó estar en desacuerdo o totalmente en desacuerdo y una porción mínima $(5,2 \%)$ expresó no estar de acuerdo ni en desacuerdo.

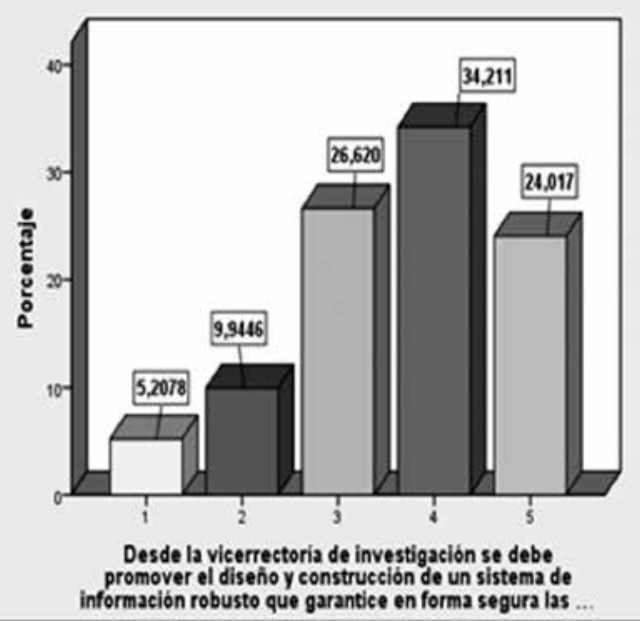

Fig. 13 Corresponde a la Pregunta No. 13 del instrumento de la encuesta.

Se concluye que la mayoría de los participantes están de acuerdo en cuanto a que es desde la Vicerrectoría de Investigación que debe nacer el diseño y construcción de una plataforma robusta que permita el almacenamiento, actualización y consulta de todo lo referente a los proyectos y productos de investigación obtenidos en el tiempo y espacio, tal como lo recomienda la gobernanza TIC en Colombia [16].

\section{F. Fase de desarrollo de las Metodologías $R U P$, con apoyo al UML, y PMBOK.}

Se tuvo en cuenta la metodología Rational Unified Process (RUP), basada en Unified Modeling
Language (UML) y el apoyo de los cinco (5) primeros grupos de proceso de PMBOK como se observa en la fig. 14: proceso de iniciación, procesos de seguimiento y control, procesos de planificación, procesos de ejecución y procesos de cierre.

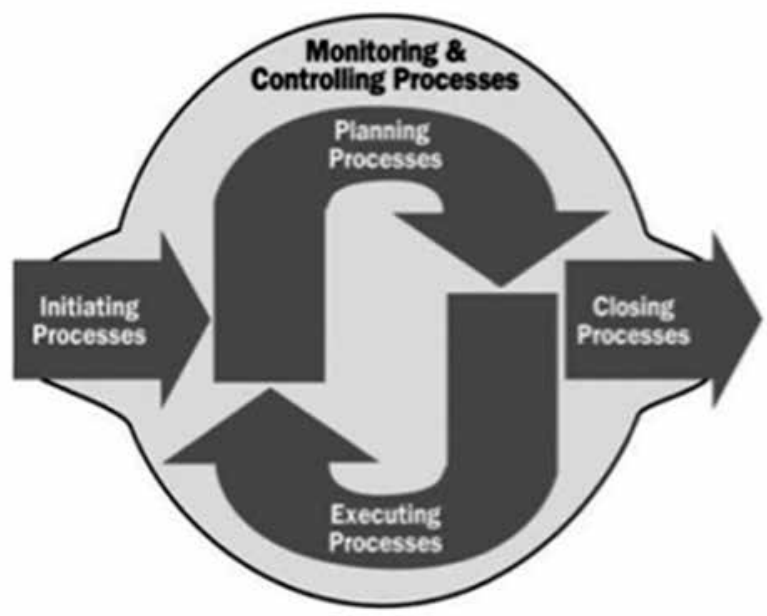

Fig. 14 Procesos de PMBOK. [14]

Para la Ingeniería de Sistemas y de Software, utilizada en el desarrollo del proyecto Geinve v1.0, acorde con lo expresado por Nextel Engineering [12], la metodología RUP, tomada para la aplicación del ciclo de vida de un proyecto, se divide en las siguientes fases:

1) Concepción. Se define el alcance del sistema, ala estimación de costos, la síntesis de la arquitectura candidata y la organización del proyecto.

2) Elaboración. Comprende las actividades para la refinación y validación de la arquitectura y los componentes.

3) Construcción. Se gestionan los recursos, optimización y control de los procesos y se completa el desarrollo de componentes.

4) Transición. Se ejecutan los planes de implementación, se terminan los manuales de usuario y técnicos, se integra el sistema y se ajusta según la validación que hace el usuario. 
Estas fases de la metodología permiten darle al desarrollo del software cierta flexibilidad, ya que trabajan a través de una serie de clases dentro del esquema general. Cada una de estas clases engloba una serie de casos de uso partiendo de los más críticos hasta los más específicos. Además, permite que cada fase se subdivida a su vez en la cantidad de iteraciones necesarias para el cumplimiento de los objetivos del proyecto Geinve-v1.0.
Lo anterior, se constituyó en una gran ventaja para el diseño del prototipo Geinve-v1.0, ya que permitió considerar, inicialmente para cada clase, solo los casos de uso más críticos que son importantes de desarrollar para aprobar la viabilidad de algún concepto en particular, iterando una o más veces de acuerdo con las necesidades del problema, para luego abordar otro ciclo que incluya nuevas problemáticas por resolver [13]. En la tabla 5 se aprecia el resultado de estas iteraciones hasta encontrar las versiones adecuadas en el desarrollo de la metodología.

TABLA V

Historial de ReVISIONES

\begin{tabular}{|c|c|c|c|}
\hline Fecha & Versión & Descripción & Autores (Semilleros) \\
\hline $15 / 10 / 2014$ & 0.9 & $\begin{array}{l}\text { Versión preliminar como propuesta } \\
\text { de desarrollo. }\end{array}$ & MENTE VIVA / APROVECHMOS LA WEB \\
\hline $05 / 12 / 2014$ & 1.0 & $\begin{array}{l}\text { Versión propuesta para aprobación } \\
\text { al final de la fase de inicio. }\end{array}$ & MENTE VIVA / APROVECHMOS LA WEB \\
\hline $21 / 02 / 2015$ & 1.9 & $\begin{array}{l}\text { Versión lista para ser revisada al fi- } \\
\text { nal de la fase de elaboración. }\end{array}$ & MENTE VIVA / APROVECHMOS LA WEB \\
\hline $18 / 03 / 2015$ & 2.0 & $\begin{array}{l}\text { Versión revisada por el Stakeholder } \\
\text { al final de la fase de elaboración. }\end{array}$ & MENTE VIVA / APROVECHMOS LA WEB \\
\hline 20/04/2015 & 2.1 & $\begin{array}{l}\text { Versión revisada en la primera ite- } \\
\text { ración de la fase de construcción. }\end{array}$ & MENTE VIVA / APROVECHMOS LA WEB \\
\hline $28 / 05 / 2015$ & 2.9 & $\begin{array}{l}\text { Versión revisada en la segunda ite- } \\
\text { ración de la fase de construcción, } \\
\text { pendiente de revisión del Stakehol- } \\
\text { der. }\end{array}$ & MENTE VIVA / APROVECHMOS LA WEB \\
\hline 27/06/2015 & 3.0 & $\begin{array}{l}\text { Versión revisada en la segunda ite- } \\
\text { ración de la fase de construcción, } \\
\text { pendiente de aprobación del Stake- } \\
\text { holder. }\end{array}$ & MENTE VIVA / APROVECHMOS LA WEB \\
\hline
\end{tabular}

En la tabla 6 se aprecia el plan de fases que se llevó a cabo durante el proceso, resaltando el tipo de clase y el hito respectivo de una manera detallada. Cabe anotar que este plan sufrió bastantes modificaciones durante su montaje debido a la madurez que se iba alcanzando en el historial de las versiones del proceso tal como se ilustra en la tabla en mención. 
TABLA VI

Plan de las Fases

\begin{tabular}{|c|c|}
\hline Descripción & Hito \\
\hline Fase de inicio & $\begin{array}{l}\text { Se desarrolló acorde con la perspectiva del equipo de } \\
\text { trabajo (semilleros Mente viva y Aprovechemos la web) } \\
\text { al igual que de los investigadores del grupo Sigcienty, } \\
\text { la cual se estableció bajo el esquema de casos de uso } \\
\text { acorde con la planeación del desarrollo del proyecto. }\end{array}$ \\
\hline Fase de elaboración & $\begin{array}{l}\text { Se analizaron los requisitos y se inició el desarrollo del } \\
\text { prototipo de arquitectura Geinve v1.0 (incluyendo las } \\
\text { partes más relevantes y / o críticas del sistema). En } \\
\text { esta fase se enmarcaron todos los casos de uso co- } \\
\text { rrespondientes a requisitos que fueron implementados } \\
\text { en la primera versión (reléase) de la fase de construc- } \\
\text { ción de la metodología en el prototipo. } \\
\text { Se logró el diseño de la estructura Geinve_v1.0 (en el } \\
\text { Modelo de Análisis / Diseño). La revisión y aceptación } \\
\text { del prototipo de la arquitectura del sistema marcó el } \\
\text { final de esta fase. }\end{array}$ \\
\hline Fase de construcción & $\begin{array}{l}\text { Se terminó de analizar y diseñar todos los casos de } \\
\text { uso, refinando el Modelo de Análisis / Diseño. El pro- } \\
\text { ducto se construyó con base en } 4 \text { iteraciones, cada } \\
\text { una produciendo una versión (reléase) a las que se } \\
\text { le aplicaron las pruebas y se validaron con el clien- } \\
\text { te/usuario. Se comenzó la elaboración de material de } \\
\text { apoyo al usuario. Al final se logró obtener un avance } \\
\text { que marcó el final de la fase en la versión ralease 4.0, la } \\
\text { cual fue entregada a los semilleros para que iniciaran } \\
\text { su proceso de prueba y ajustes. }\end{array}$ \\
\hline Fase de transición & $\begin{array}{l}\text { Se prepararon dos raleases para distribución, asegu- } \\
\text { rando una implantación y cambio del sistema previo de } \\
\text { manera adecuada, incluyendo el entrenamiento de los } \\
\text { usuarios. El proceso que marcó el fin de esta fase in- } \\
\text { cluye la entrega de toda la documentación del proyecto } \\
\text { con los manuales de instalación y todo el material de } \\
\text { apoyo al usuario, la finalización de la capacitación de } \\
\text { los usuarios y el empaquetamiento del producto. }\end{array}$ \\
\hline
\end{tabular}

\section{G. Iteraciones de la metodología (RUP).}

A continuación se presenta un calendario de las principales tareas del proyecto incluyendo las cuatro fases de la metodología RUP. Se debe tener presente que el proceso iterativo e incremental de RUP se caracteriza por la realización en paralelo de todas las disciplinas de desarrollo a lo largo del proyecto, con lo cual la mayoría de los artefactos se generan muy tempranamente, pero se van llevando a cabo en mayor o menor grado de acuerdo con la fase e iteración del mismo. La fig. 15 ilustra este enfoque de las fases. Lo ensombrecido marca el énfasis de cada disciplina (workflow) en un momento determinado del desarrollo. 


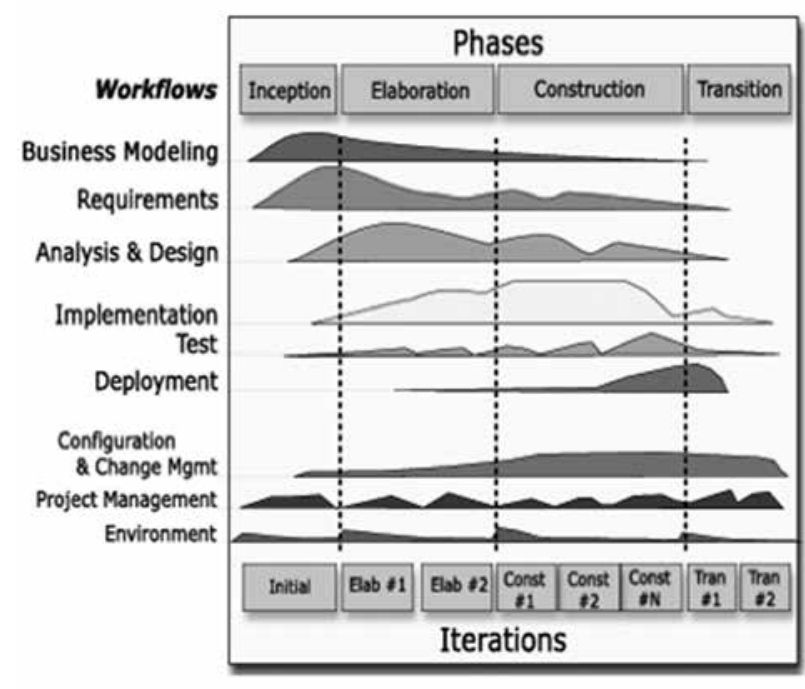

Fig. 15 Fases de la metodología RUP

Así mismo, se estableció un cronograma de trabajo, con una fecha de aprobación de los procesos y productos que indica cuándo está completamente listo el producto para someterse a revisión y aprobación; sin embargo, durante su ejecución se hicieron algunas actualizaciones acordes con los objetivos planteados en el proyecto.

\section{H. Aplicación de las áreas de conocimiento de PMBOK.}

Una vez establecida la metodología RUP con los cinco (5) grupos de procesos PMBOK, se continuó con las nueve (9) áreas de conocimiento de PMBOK [14] con el fin de lograr completar la parte de negocios del prototipo Geinve v1.0.

Estas áreas se pueden apreciar en la figura 16: 1) Gestión de la Integración del Proyecto: incluye los procesos y actividades necesarios para identificar, definir, combinar, unificar y coordinar los diversos procesos y actividades de la dirección de proyectos dentro de los grupos de procesos de dirección de proyectos; 2) Gestión del alcance del proyecto: incluye los procesos necesarios para garantizar que el proyecto contenga únicamente lo necesario para completarla con éxito; 3) Gestión del tiempo del proyecto: incluye los procesos requeridos para administrar la finalización del proyecto a tiempo; 4) Gestión de los costos del proyecto: incluye los procesos involucrados en estimar, presupuestar y controlar los costos de modo que se complete el proyecto dentro del presupuesto aprobado; 5)Gestión de la calidad del proyecto: incluye los procesos y actividades de la organización ejecutante que determinan responsabilidades, objetivos y políticas de calidad a fin de que el proyecto satisfaga las necesidades por la cuales fue emprendido; 6) Gestión de los recursos humanos del proyecto: incluye los procesos que organizan, gestionan y conducen el equipo del proyecto; 7) Gestión de las comunicaciones del proyecto: incluye los procesos requeridos para garantizar que la generación, la recopilación, la distribución, el almacenamiento, la recuperación y la disposición final de la información del proyecto sean adecuados, oportunos, y entregada a quien corresponda (interesados del proyecto o stakeholders); 8) Gestión de los riesgos del proyecto: incluye los procesos relacionados con la planificación de la gestión, identificación, el análisis, la planificación de respuesta a los riesgos, así como su monitoreo y control en un proyecto y 9) Gestión de las adquisiciones del proyecto: incluye los procesos de compra o adquisición de los productos, servicios o resultados que necesariamente se deben obtener fuera del equipo del proyecto [14]. 


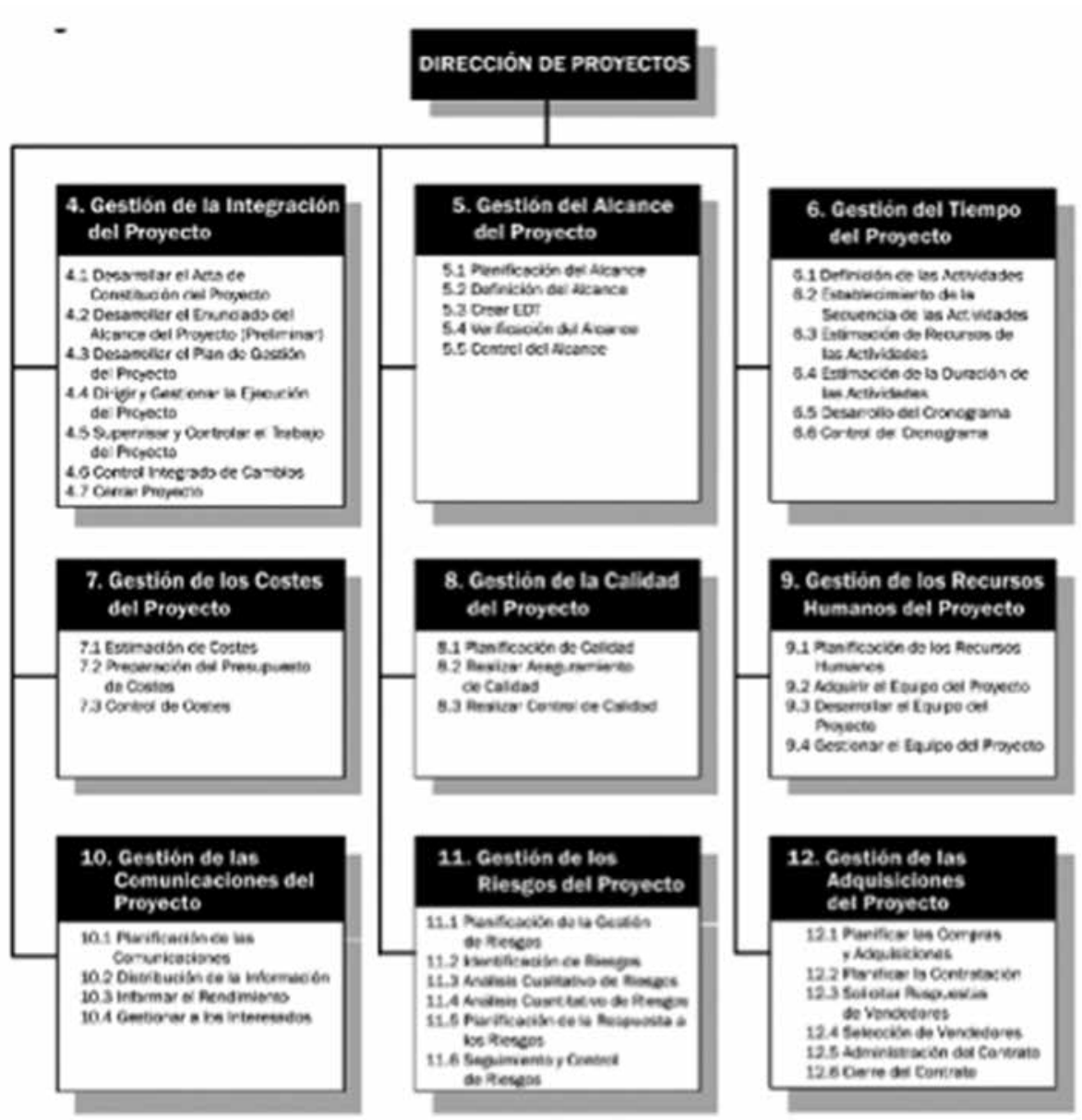

Fig. 16 Áreas de conocimiento de PMBOK aplicadas en Geinve v1.0

Para cada una de estas áreas de gestión se realizó su respectivo cronograma de trabajo resaltando el tiempo, porcentaje de avance y responsabilidades, siendo esta la parte más compleja del estudio debido a la poca experiencia de los encargados del esquema de negocios. Sin embargo, se logró articular el enfoque de casos de usos, con las partes de la metodología RUP.

\section{Prototipo Geinve-v1.0.}

Propone una estructura adaptada a la metodología RUP /PMBOK, conformada por aspectos dinámicos y estáticos. Las fases e hitos corresponden a los aspectos dinámicos, y las disciplinas, a los aspectos estáticos. Estas metodologías permiten la adaptación de los procesos, es decir, son marcos de trabajo que facilitan la selección de elementos según las características de cada proyecto, lo cual permitió definir la metodología Geinve v1.0 con un enfoque de calidad y con base en las necesidades de los proyectos de investigación de la Universidad ECCl.

\section{J. Estructura dinámica de la metodología.}

El prototipo Geinve v1.0. Interactúa a través de un nivel repetitivo a lo largo de una serie de ciclos que constituyen la vida de un proyecto. Cada ciclo concluye con una generación del producto y consta de cuatro fases, cada una de las cuales se subdivide, a la vez, en iteraciones; el número de iteraciones en cada fase es variable. Cada fase concluye con un hito bien definido; un punto en el tiempo en el cual se deben tomar ciertas decisiones críticas y alcanzar la meta clave antes de pasar a la siguiente fase. Ese hito principal de cada fase se compone de hitos menores que podrían 
ser los criterios aplicables a cada iteración. Los hitos son puntos de control sobre los cuales los involucrados en el proyecto revisan el progreso del mismo.

El ciclo de vida propuesto por la metodología Geinve v1.0 para un proyecto de investigación se compone de cuatro fases secuenciales que son: inicio, elaboración, construcción y transición. Al final de cada fase, el director o líder de proyecto realiza una evaluación para determinar si los objetivos se cumplieron y si se puede pasar a la fase siguiente.

\section{K. Estructura estática de la metodología.}

Una metodología de evaluación define quién hace qué, cómo y cuándo. Para tal acción la metodologia Geinve v1.0 define cuatro elementos: los roles, que responden a la pregunta ¿quién?; las actividades, que responden a la pregunta ¿cómo?; los artefactos, que responden a la pregunta ¿qué? y los flujos de trabajo de las disciplinas que responden a la pregunta ¿cuándo producir artefactos?

Las disciplinas que conforman esta metodología se dividen en dos grupos. El primero comprende las disciplinas fundamentales asociadas con las áreas de Ingeniería: modelado empresarial, requisitos, análisis y diseño, implementación, pruebas y despliegue.

El segundo grupo lo integran las disciplinas conocidas como de soporte o gestión: gestión del proyecto y gestión de cambios.

\section{Artefactos.}

La metodología Geinve-v1.0 consta de veinticuatro (24) artefactos en total, divididos en cada una de sus disciplinas. Cabe anotar, que los artefactos se aplican dependiendo del tamaño y alcance de los proyecto [14].

\section{1) Proyectos de investigación.}

Requisitos (fase de inicio):

- Casos de uso.

- Documento de requisitos del sistema DRS.

- Glosario.

- Solicitudes de los interesados.

- Visión del sistema.

Análisis y diseño (fase de elaboración):

- Documento de arquitectura de software DAS.

Implementación (fase de construcción)

- Registro de pruebas unitarias.

Pruebas (fase de construcción):

- Ideas de prueba.

Gestión del proyecto (todas las fases):

- Acta de reunión.

- Gráfica del trabajo pendiente.

- Lista de elementos de trabajo.

- Plan del proyecto.

- Registro de revisión.

- BPMN

Gestión de cambios (fase de transición):

- Solicitud de cambio.

\section{2) Proyectos complejos:}

Modelado de negocio (fase de inicio):

- Organigrama

- Modelo de procesos.

Requisitos (fase de inicio):

- Casos de uso.

- Documento de requisitos del sistema DRS.

- Glosario

- Solicitudes de los interesados.

- Visión del sistema. 
Análisis y diseño (fase de elaboración):

- Decisiones de arquitectura.

- Documento de arquitectura de software DAS.

Implementación (fase de construcción):

- Registro de pruebas unitarias.

Pruebas (fase de construcción):

- Casos de pruebas.

- Ideas de prueba.

- Lista de ideas de prueba.

Gestión del proyecto (todas las fases):

- Plan de gestión de comunicaciones.

- Plan de gestión de riesgos.

- Acta de entrega del proyecto.

- Acta de reunión.

- Gráfica del trabajo pendiente.

- Lista de elementos de trabajo.

- Plan del proyecto.

- Registro de revisión.

- Informe de avance.

- Plan de iteración.

- BPMN.

Gestión de cambios (fase de transición):

- Solicitud de cambio.

\section{Discusión de Resultados}

\section{A. Similitud de Geinve v1.0 con otros proto- tipos}

En el ámbito latinoamericano se destaca la experiencia de la Universidad Mayor de San Simón en Bolivia la que, en conjunto con la Agencia Sueca para el Desarrollo Internacional (ASDI), se propuso en el año 2003 llevar a cabo un Programa de Cooperación de la Investigación Científica, siendo uno de sus resultados el diseño de un sistema para fortalecer la gestión de las actividades de investigación en dicha universidad. Lo mismo ocurre con el sistema creado en la Universidad Católica de Colombia, la cual ha optado por constituir un Sistema de Investigaciones de nivel central que integra las instancias académicas, administrativas y de gestión de la institución, en torno a la investigación [15]. El historial investigativo de estas universidades se asemeja un poco al de la Universidad ECCI en el sentido que desde hace seis años le ha asignado una especial relevancia al quehacer investigativo [3].

Una revisión de los módulos que incluye cada uno de los sistemas da cuenta de una fuerte similitud entre los módulos del prototipo Geinve-v1.0 aunque las metodologías PMBOK y RUP / UML son especiales de Geinve-v1.0, ya que describen paso a paso la interactividad-entre el proceso y la fase en la gestión del proyecto logrando que los usuario se familiaricen e interactúen de una forma sencilla con el procedimiento por seguir en cada módulo.

El proceso de elaboración del Proyecto Geinve-v1.0, se enfocó en el esfuerzo de los grupos semilleros Mente viva y Aprovechemos la web, apadrinados por el grupo de investigación Sigcienty para construir los elementos críticos estructuralmente y del comportamiento de los Ilamados elementos arquitecturales antes de construir elementos menos importantes, con la exploración y comprensión de las metodologías bases como fueron PMBOK y RUP [15], [16].

\section{B. Motivación en la comunidad}

La construcción del prototipo del proyecto Geinve-v1.0 en la Universidad ECCI contribuye a motivar a sus actores (estudiantes, docentes, investigadores y demás) a incrementar significativamente la producción científica, académica, tecnológica e innovadora ya que encuentran un sitio web seguro y confiable en el que se puede generar un proyecto de investigación y realizar el seguimiento continuo del mismo hasta obtener sus productos, garantizando la alta calidad para la gestión del conocimiento en la comunidad científica del país.

Geinve-v1.0 se basa en estrategias de mejoramiento continuo en las que está empeñada la institución, con miras a optimizar su competitividad 
agregando más valor a sus procesos internos de investigación; aumentar la eficiencia con que se realizan sus actividades; mejorar su productividad y reducir sus costos. Esta es una de las estrategias que hará posible aumentar la cantidad y calidad de las investigaciones realizadas en la Institución.

La gestión investigativa en la Universidad ECCI se verá significativamente beneficiada con el desarrollo de Geinve-v1.0 (prototipo funcional fase inicial - de tres capas). Además, como sistema informático también tiene grandes desafíos para el futuro. El activar algunos módulos de Geinve-v1.0 en la plataforma virtual de aprendizaje de los estudiantes de la Institución para la selección de algunos proyectos de investigación, seminarios, pasantías o envío de propuestas, genera gran expectativa en esta comunidad logrando la continuidad y terminación de proyectos, entre otros, analizar y evaluar la propuesta enmarcada en el esquema oficinal de la Vicerrectoría de Investigación.

\section{Impacto sobre la productividad y competitividad}

El uso de las herramientas y los productos derivados del proyecto permitirá potenciar las capacidades de los jóvenes investigadores y sus semilleros de investigación de la Facultad de Ingeniería, en cuanto a la formación tecnológica de metodologías estándares evaluadas por prototipos para el desarrollo de sistemas de información de carácter específico. Estas herramientas a futuro permitirán mejorar los métodos de aprendizaje en la construcción de modelos y prototipos funcionales que faciliten conocer de manera rápida y precisa las entradas, procesos y salidas en el ciclo de vida de un sistema de información (producción de software) [17].

\section{Impacto sobre el medio ambiente y la sociedad}

En relación con el impacto social, la aplicación en gran parte de metodologías reconocidas y exitosas le ofrece al prototipo funcional una gran oportunidad para que en un futuro sea utilizado como guía para generar sistemas rápidos y seguros de negocios y generación de software para todo tipo de población [17]. Este tipo de herramientas tecnológicas blandas con el apoyo de las metodologías podrían más adelante llegar a minimizar considerablemente los errores en los sistemas de información grandes y sobre todo garantizar el funcionamiento óptimo de los mismos, sin importar las variables exógenas que los regulen, contribuyendo al esquema de negocios al interior de la institución, y mejorando así la apropiación tecnológica e innovadora de las buenas prácticas en los procesos de organización y clasificación de la información.

\section{E. Impactos de ciencia, tecnología o innovación}

El uso de las metodologías estándares y sus bondades en la construcción de prototipos funcionales son alternativas de fácil diseño y de una gran operabilidad en su funcionamiento, garantizado los puntos fuertes del sistemas y controlando los errores y ajustes del mismo, hasta encontrar su punto óptimo. Esto, sin ninguna duda, tiende a mejorar los grandes sistemas de información en las diferentes áreas del conocimiento, garantizando la optimización de los mismos. El manejo de un prototipo funcional para la gestión investigativa abre las puertas a las instituciones de educación superior para trabajar en sus direcciones o vicerrectorías con un esquema de proyectos de investigación [17]. Esto genera bastante impacto en la comunidad dado que las entidades públicas y privadas, como también el público en general, muestran cierto interés de acercarse a productos tecnológicos cuando estos hacen parte de la solución a problemas reales y, en particular, cuando están relacionados con sistemas que pueden contribuir a reducir los altos índices de robo de información o pérdida de la misma, tema de profundo interés en nuestra sociedad. 


\section{F. Otros impactos}

1) Corto plazo. El desarrollo de este proyecto surge como una nueva alternativa para la realización, por una parte del Diseño de un sistema de información para la gestión investigativa en las universidades colombianas, tomando como prototipo la Universidad ECCI, con el apoyo de metodologías reconocidas y exitosas. $\mathrm{Y}$, por otra, de novedosas plataformas de interacción (aplicación web y aplicación para dispositivos móvil), cuyos avances tecnológicos permiten afianzar los conocimientos del personal científico [16].

La utilización del sistema aquí propuesto resulta una alternativa idónea debido al alto impacto de innovación y tecnología. Asimismo, se generará nuevo conocimiento en Educación en Ingeniería y Desarrollo Tecnológico y en el área de modelaje específico para soluciones reales.

2) Largo plazo. Se requiere continuar con el desarrollo del prototipo en el sistema de información para la gestión investigativa, con el fin de lograr la conformación del sistema integral de información y así contribuir significativamente con la generación de nuevas técnicas para el control y manejo de la producción científica y específica hacia las direcciones de los centros de investigación en las universidades, con mayores funcionalidades, integrados con metodologías, negocios, método y desarrollo ingenieril.

3) Mediano plazo. El desarrollo de este proyecto sienta las bases para el surgimiento de nuevas propuestas conducentes a la integración de nuevas tecnologías, metodologías, sistemas y dispositivos que contribuyan a la línea de investigación en Ingeniería y Desarrollo Tecnológico. Dichas bases posibilitan el surgimiento de nuevas propuestas en este campo, así como también garantizan la continuidad de esta línea de investigación en los grupos involucrados, y el fortalecimiento de los laboratorios de desarrollo de software con una visión de negocio en la producción investigativa en las universidades, en especial la Universidad ECCI.
La presentación de todo el esquema del prototipo Geinve-v1.0, se encuentra en el libro de investigación: Diseño del prototipo para el Sistema de Información del Modelo Integral en la Gestión Investigativa de la Universidad ECCI, elaborado en junio del 2015 y próximo a publicarse. En la figura 17 se observa la pantalla principal de dicho prototipo identificando algunos de sus módulos como: grupos de investigación, proyectos de investigacion, semilleros de investigacion, repositorio documental, y ficha proyectos de investigacion entre otros.

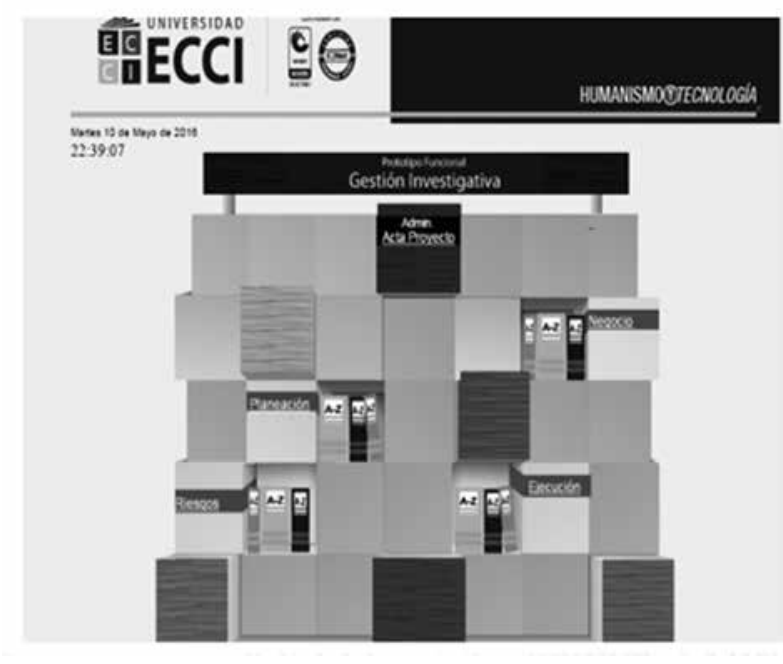

Fig. 17 Esquema digital del prototipo GEINVE V1.0 [18]

\section{Conclusiones}

El prototipo Geinve-v1.0 contribuye en gran parte a fortalecer los procesos y procedimientos al interior de la Vicerrectoría de Investigación de la Universidad $\mathrm{ECCl}$, por su fácil manejo y sobre todo por costos mínimos de implantación en su primera fase. Geinve-v1.0 está diseñado con herramientas libres como MySQL, JSP con el IDE de NETBEENS ver. 8.2, bajo la licencia de GNU. Su instalación y puesta en marcha está configurada para trabajar con dos servidores (máquinas): uno para la base de datos (MySQL) y el otro para el aplicativo (TOMCAT). Esta configuración obedece 
a proteger los datos y extender en un alto grado de seguridad las transacciones en línea (web) del sistema [16], [17].

El ciclo de vida de un proceso de desarrollo en Geinve-v1.0 consiste en el apoyo bajo la metodología RUP que consta de cuatro fases: inicio, elaboración, construcción y transición. Cada fase puede incorporar una o varias iteraciones, en las que se realizan actividades de aseguramiento de calidad y mitigación de riesgos, de manera continua.

La utilización del prototipo Geinve-v1.0 por parte de los investigadores, permitirá mejorar la capacidad de adopción y adaptación de un proceso de desarrollo para la ejecución de sus proyectos de investigación, estableciendo una línea de tiempo entre las actividades y los productos esperados.

\section{Reconocimientos}

Los investigadores del grupo Sigcienty agradecen especialmente a los estudiantes de los semilleros Mente viva y Aprovechando la web, por su gran dedicación y esfuerzo en el desarrollo de este proyecto, como aporte al inicio de una investigación aplicada al interior de la Universidad ECCl.

\section{Referencias}

[1] J. F. López, Plan Estratégico de la Vicerrectoría de Investigación. Escuela Colombiana de Carreras Industriales. Bogotá, Colombia: Ed. ECCI, 2012.

[2] Colciencias, Grupo Publindex, Estrategia para mejorar la calidad y cantidad de las revistas científicas colombianas. Bogotá, D.C. 2010.

[3] N. Ochoa, Estado del arte: El modelo de evaluación de investigación en las universidades Latinoamérica. Bogotá, Colombia: Ed. ECCI. http://revistasdm. ecci.edu.co/index/IngECCI. 2012.
[4] M. C. J. Caniëls and R. J. J. M. Bakens, "The effects Of Project Management Information Systems on decision making in a multi project environment," International Journal of Project Management, vol. 30, No. 2,. 2010, pp. 162-175.

[5] Anderson L. O. El modelado del negocio utilizando la metodología Rational Unified Process (RUP). 2013.

[6] D. J. Rodríguez, Software educativo para la enseñanza-aprendizaje del Psicodiagnóstico de Rorschach. Autor: Sagua la Grande. Villa Clara. Cuba. Universitaria de Ciencias Médicas "Lidia Doce Sánchez", 2012.

[7] Parra C., Ruiz R., Pérez P. Modelado de procesos y desarrollo de sistemas software: integración entre UML y EPC. Sevilla España. 2012.[8] V. D. Rada, Análisis de datos de encuestas, Barcelona, España, 2011, pp 19-29.

[8] N. Malhotra, Investigacion de Mercados: Un enfoque aplicado (4ta. Edición). México: Prentice Hall, 2010.

[9] R. Hernández, C. Fernández, P. Baptista, Metodología de la investigación. 6ta. edición. México, McGraw-Hill. 2006.

[10] C. H. Lawshe, "Modelo para el dictamen cuantitativo de la validez de contenido de un instrumento". 28(4), 563-575. 2008.

[11] PMI, A Guide to the Project Management Body of Knowledge ( $P M B O K^{\circledR}$ Guide ), Fifth Edit. Newton Square, Pennsylvania USA: Project Management Institute Inc, 2013.

[12] I. Jacobson, G. Booch, J. Rumbaugh El Proceso Unificado de Desarrollo de Software. Sexta edición. México D:F:, Editorial McGraw Hill, 2010.

[13] C. Franco, (2009, Ene 11) Prácticas expuestas en el PMBOK. [Online]. Available: http://blog.pucp.edu.pe/ blog/franco/2009/01/11/pmbok. 2009.

[14] H. Ferreyra, "Construir futuros posibles: el desafío de aprender a emprender en las sociedades del conocimiento. Tiempo de Educar" 12(23), 9-28. 2011

[15] ISO, "ISO/IEC 38500:2015 Information technology Governance of IT for the organization Technologies de l'information - Gouvernance des technologies de l'information pour l'entreprise (Second Edition 201502-15 ed.). Technical report," 2015.

[16] P. L. Bannerman, "Software development governance: A meta-management perspective," Softw. Dev. Governance, 2009. SDG '09. ICSE Work., no. IEEE, pp. 3-8, 2009.

[17] SIGCIENTY. 2015, Jun 30. Apache. Universidad ECCI. [Online]. http://localhost:8080/GEINVE_V1/ 